\title{
PERFIL DE CRIANÇAS VÍTIMAS DE ACIDENTE MOTOCICLÍSTICO INTERNADAS EM HOSPITAL REFERÊNCIA EM TRAUMA NO ESTADO DO PARÁ: região amazônica
}

Juliana Anezia Rodrigues do AMARAL ${ }^{1}$

Eduardo do Carmo SILVA ${ }^{2}$

Fabiano José da Silva BOULHOSA ${ }^{3}$

Gabriela Martins LIMA ${ }^{4}$

Kéven Lorena de Paula GONÇALVES ${ }^{5}$

Leonardo Ramos Nicolau da COSTA ${ }^{6}$

Paola Katherine Esteves da SILVA ${ }^{7}$

Patrícia Gazel PICANÇO ${ }^{8}$

Rafaela Cordeiro de MACÊDO 9

Renato da Costa TEIXEIRA ${ }^{10}$

${ }^{1}$ Especialista em Urgência e Emergência no Trauma - Universidade do Estado do Pará; email: julianadoamaral@ymail.com

${ }^{2}$ Acadêmico do $10^{\circ}$ período de medicina do Centro Universitário do Pará; email: eduardo.carmo.silva@ hotmail.com

${ }^{3}$ Especialista, preceptor do Programa de Residência Multiprofissional em Saúde em Urgência e Emergência no Trauma

- Universidade do Estado do Pará; email: fabiano.boulhosa@gmail.com

${ }^{4}$ Especialista, Coordenadora do Setor de Reabilitação do Hospital Metropolitano de Urgência e Emergência; email:gabimlima@gmail.com

${ }^{5}$ Especialista, preceptor do Programa de Residência Multiprofissional em Saúde em Urgência e Emergência no Trauma

- Universidade do Estado do Pará; email: keven.lorena@ hotmail.com

${ }^{6}$ Mestre, coordenador do Programa de Residência Multiprofissional em Saúde em Urgência e Emergência no Trauma Universidade do Estado do Pará; email: leorcosta@gmail.com

${ }^{7}$ Fisioterapeuta Residente do Programa de Residência Multiprofissional em Saúde em Urgência e Emergência no Trauma- Universidade do Estado do Pará; email: paolaestevessilva@ yahoo.com

${ }^{8}$ Fisioterapeuta Residente do Programa de Residência Multiprofissional em Saúde em Urgência e Emergência no Trauma- Universidade do Estado do Pará; email: patricia_gazel@ hotmail.com

${ }^{9}$ Especialista, preceptor do Programa de Residência Multiprofissional em Saúde em Urgência e Emergência no Trauma

- Universidade do Estado do Pará; email: rafaela_cmacedo@ @otmail.com

${ }^{10}$ Doutor, professor adjunto III da Universidade do Estado do Pará; email: teixeirarenato @ globo.com

Recebido em: 17/11/2015 - Aprovado em: 13/07/2016 - Disponibilizado em: 18/12/2016 
RESUMO: Traumas decorrentes de acidentes de motocicleta é uma importante causa de morbi-mortalidade em crianças, principalmente em países subdesenvolvidos. Neste estudo,objetivamostraçar o perfil clínico e epidemiológico de crianças internadas vítimas de acidente de moto em um hospital referência em trauma na região Amazônica. Foi conduzida de forma tipo descritiva, retrospectiva, estatística e delineou o perfil de crianças de 0 a 14 anos vítimas de acidente de moto internadas no local.Como resultados, foi encontrado que ascrianças vítimas de acidente motociclístico eram em sua maioria do sexo masculino, com idade de 2 e 14 anos, provenientes de fora da região metropolitana, vítimas de atropelamento ou conduzindo motocicletas, vítimas de TCE's ou fraturas. Uma pequena parcela necessitou de internação de UTI e uso de VMI. O tempo de internação foi considerado curto e poucas crianças evoluíram à óbito. Conclui-se que os traumas por acidentes de motocicletas são uma importante causa de internações em crianças.

Palavras-Chave: Criança. Traumatismos encefálicos. Causas externas. Acidentes. Perfil de Saúde

ABSTRACT: Trauma resulting from motorcycle accidents is a major cause of morbidity and mortality in children, especially in underdeveloped countries. In this study, we aimed to establish a clinical and epidemiological profile of children hospitalized victims of motorcycle accidentsin a reference trauma hospital in the Amazon region. It was conducted in a descriptive way, retrospective, statistics and outlined the profile of children aged 0 to 14 years, motorcycle crash victims admitted in the hospital. As a result, it was found that children victims of motorcycle accidents were mostly male, aged 2 to 14 years, from outside the metropolitan area, victims of trampling or driving motorcycles, traumatic brain injury's victims or fractures. A small portion required ICU admission and use of invasive mechanical ventilation. The length of stay was considered short and few children have evolved to death. It concludes that trauma by motorcycle accidents are an important cause of hospitalizations in children.

Keywords:Child. Brain injuries.External causes.Accidents. Health Profile.

\section{INTRODUÇÃO}

Por ano, ocorrem cerca de um milhão de mortes na população até 14 anos, decorrentes de acidentes no trânsito no mundo, onde mais de $90 \%$ acontecem em países em desenvolvimento. Os acidentes de trânsito não são obras do acaso, e sim resultado de possíveis erros e falhas (PEREIRA et al., 2011).

No Brasil, de 2000 à 2010,a segunda maior causa de internações por causas externas foramos acidentes de transporte terreste, em que houve aumento de $8,7 \%$ no risco de internação no período citado. Por outro lado, analisando-se o tipo de vítima nos acidentes de transporte terrrestes, o risco de internações de pedestres descresceu, enquanto houve um aumento de $243,1 \%$ no risco de internação para acidentes com motocicleta (MASCARENHAS et al, 2013).

Mesmo que os pedestres somem a maioria dos vitimados nos acidentes de trânsito, os óbitos relacionados à motocicletas tem ocorrido em proporção exponencial. Proporcionalmente, as vendas de motocicletas crescem consideravelmente a cada ano. Acredita-se que o crescimento da frota de motocicleta ocorre pelo fato de ser um meio de transporte rápido que propicia uma facilidade no deslocamento em congestionamentos, por ser um veículo econômico, pela facilidade de estacionamento, bem como pelo crescimento do mercado de serviços viabilizados pela moto. Por outro lado, os mesmos fatores são responsabilizados pelo uso indiscriminado, sendo a facilidade de aquisição um fator que pode propiciar os próprios acidentes 
(GOLIAS, CAETANO, 2013; SANT'ANNA

et al., 2013).

Um fator que deve ser considerado como crítico é quando esses traumas acontecem em crianças ou menores em desenvolvimento, pelas sequelas que podem ser instaladas. No estudo de Figueiredo Júnior, Carvalho e Lima (2012) que analisou os atendimentos por trauma pediátrico, observou que $71,15 \%$ das crianças menores que 12 anos foram vítimas de acidentes de trânsito, ilustrando assim, o grau de influência destes acidentes nas questões que inferem ao traumatismo da criança.

O mundo da criança se estende da casa para a escola e outras atividades sociais e assim, eles se tornam também susceptíveis à acidentes de trânsito, pois aumentam o uso de vias públicas seja como pedestres ou ciclistas, motociclistas e passageiros de veículos. Porém, o sistema de transporte muito raramente é projetado visando estes menores, devendo ser considerados uma população vulnerável. Menores que fazem o uso de motocicleta estão iminentemente em risco pois, em caso de acidente, estão mais propensos a grandes lesões (OXLEY et al., 2013).

No entanto, a criança, durante seu crescimento, ainda está desenvolvendo os fatores decisivos para o discernimento no trânsito, o que a deixa mais susceptível a se tornarem vítimas desse tipo de acidente, além de sua menor estatura que prejudica a visualização dos condutores (FREITAS; RIBEIRO; JORGE, 2007).

A falta de maturidade em seguir as leis do trânsito das crianças condutoras de motocicletas, somado à impunidade, à negligência ao uso de equipamentos de segurança são fatores que propiciam os acidentes e traumas nestes menores (JORGE; MARTINS, 2013).

Jorge e Martins (2013) frisam que quando a criança for maior de 7 anos e puder ser transportada, o condutor deve tomar atenção redobrada, usando sempre os equipamentos de segurança, uma vez que em caso de acidente, o trauma na criança tende a ser mais complicado que em um adulto.

Nos países desenvolvidos, a redução das mortes nos últimos 30 anos pode ser resultado do investimento nos pontos deficitários citados anteriormente, aliado à melhoria de ruas e estradas, campanhas educativas para trânsito, e notificação na legislação (PEREIRA et al., 2011). Assim, fica claro que somente ações articuladadas de entidades públicas e privadas que englobem a prevenção e a educação no trânsito, podem reduzir as lesões causadas em vítimas de acidentes envolvendo motocicletas (OLIVEIRA e SOUSA, 2012). Assim, este estudo visou traçar o perfil clínico e epidemiológico de crianças internadas vítimas de acidente de moto no Hospital Metropolitano de Urgência e Emergência 
(HMUE) no período de janeiro de 2010 à dezembro de 2013.

\section{MÉTODOS}

Este estudo é do tipo observacional, descritivo, retrospectivo,estatístico, em um único centro, local, sem financiamento, realizado através de análise de prontuário,quedelineou o perfil de crianças de 0 a14anos vítimas de acidente de moto internadas em um hospital referência em urgência e emergência localizado na região metropolitana de Belém do Pará.Foramincluídos neste trabalhotodos os prontuários decrianças vítimas de acidente envolvendo moto, internadas em hospital de referência na faixa etária de 0 a 14 anos no período de janeiro de 2010à dezembro de 2013.

Foramexcluídos do presente estudo prontuários que não continham todos os dados estipulados nos objetivos da pesquisa e os prontuários de crianças vítimas de outros acidentes que não seja de trânsito.Com base em um protocolo de questionário no qual foram colhidas informações sobre o perfil demográfico (sexo, idade, procedência); perfil clínico, no qual será visado conhecer quais os tipos frequentes de traumas (traumatismo crânioencefálico, fraturas, traumatorácico). As regiões acometidas do corpo foram divididas de acordo com a Classificação Internacional de Funcionalidade,
Incapacidade e Saúde (CIF). Foram avaliados também o tempo de internação, necessidade de internação em unidade de terapia intensiva (UTI) e e uso de ventilação mecânica (VM)bem como informações sobre complicações ou comorbidades decorrentes do processo de hospitalização (infecções, disfunções respiratórias, etc.). Além de informações sobre o acidente (se o menor internado era motociclista, pedestre ou ciclista).

Os dados foram armazenados em tabelas, no programa Microsoft Excel 2010®, e posteriormente foram analisadas estatisticamente através do programa Bioestat 5.3 ®. Foi feito correlação entre as variáveis fratura e tipo de tratamento e entre TCE e tipo de tratamento e internação na UTI e necessidade de VMI, sendo as variáveis nominais inicialmente transformadas em variáveis categóricas e posteriormente submetidas a analise pela correlação. Em relação as demais variáveis, foi utilizado teste de aderência do Qui-quadrado para uma amostra com proporções esperadas iguais obedecendo sempre o nível de significância de $\mathrm{p} \leq 0,05 \%$.

\section{RESULTADOS}

No presente estudo foram selecionados 472 prontuários pelo Serviço de Arquivo Médico e Estatístico. Destes, 156 $(33,05 \%)$ foram excluídos por não obdecerem 
aos critérios de inclusão.Já em relação ao tipo de acidente, nota-se que os principais tipos de vítimas foram pedestres atropelados $-n=164$ (tabela 1)-, seguida de crianças motociclistas (condutores e passageiro), $\mathrm{n}=141$ vítimas de acidente $(\mathrm{p}<0,0001)$. Atropelamentos de ciclistas ocorrem em menor quantidade correspondendo a apenas $3,48 \%$ dos acidentes.

Tabela 1 - Distribuição de menores internados no HMUE por acidente de motocicletas de acordo com o tipo de vítima.

\begin{tabular}{ccc}
\hline Tipo de acidente & $\mathrm{N}$ & $\%$ \\
\hline Pedestre & 164 & 51,89 \\
Motociclista & 141 & 44,62 \\
Ciclista & 11 & 3,48 \\
\hline
\end{tabular}

Fonte: Dados da Pesquisa

No que diz respeito aos dados demográficos, observamos

uma predominância de acidentes envolvendo crianças do sexo masculino com 62,66\% (p < 0,0001). Entre as idades, observamos que as idades de $2(n=34)$ e $14(n=36)$ anos estão mais expostas $(\mathrm{p}<0,0001)$ à sofrerem acidentes de trânsito envolvendo motocicletas.No que diz respeito ao período estudado (gráfico 1), observou-se que houve um declínio das internações de menores até 14 anos por acidente envolvendo motocicletas de 2010 a 2011, mantendo os valores próximos em 2012, seguido de uma ascensão em 2013 ( $p<0,0001)$. Quando foi analisado a distribuição por meses observou-se que não houve diferença entre os meses estudados $(\mathrm{p}>0,05)$.

Gráfico1- Evolução das interações de menores vítimas de acidente de motocicleta no período estudado.

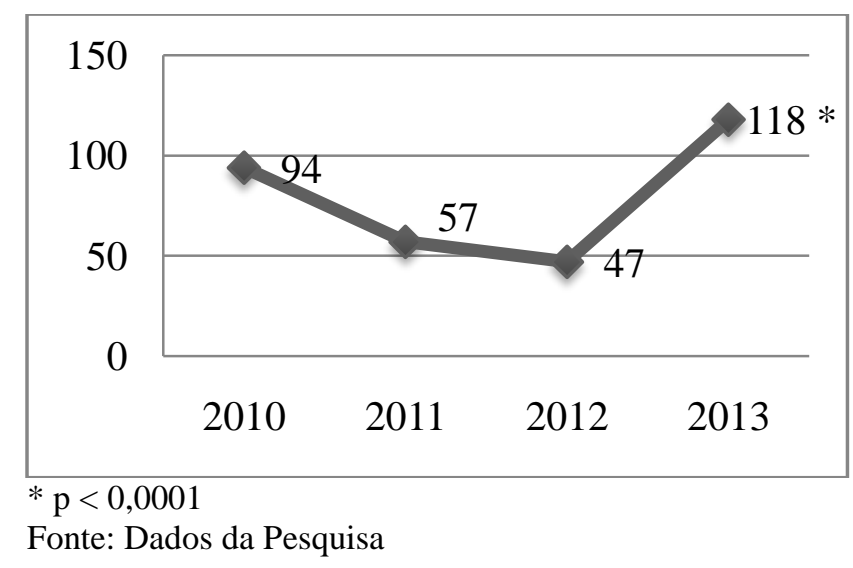

No que diz respeito à procedência, foi observado que a maioria dos indivíduos estudados $\quad(n=219, \quad 69,31 \%) \quad$ eram provenientes de cidades fora da região metropolitana de Belém ( $\mathrm{p}<0,0001)$. Com relação ao tipo de trauma, as fraturas e os traumatismoscranioencefálicos

(TCE) corresponderam a grande maioria dos traumas $(\mathrm{p}<0,0001)$ que acometeram $41,47 \%$ e $37,4 \%$ das crianças estudadas, respectivamente (gráfico 2). Já entre as estruturas do corpo, divididas pela Classificação Internacional Incapacidade e Saúde (CIF), atabela 2 mostra que as regiões mais acometidas foram as estruturas de cabeça e pescoço $(n=170)$ e estruturas dos membros inferiores $(n=167)$ de um total de 397 lesões ( $\mathrm{p}<0,0001)$. Quando verificou-seas correlações entre fraturas e tipo de tratamento, notou-se uma correlação moderada pelo Coeficiente de Pearson ( $\mathrm{r}=-$ 0,4738), constando assim que há relação entre 
as fraturas e o tratamento cirúrgico dessas crianças. Quando se analisou a relação entre TCE e o tipo de tratamento, também foi observada uma correlação moderada pelo Coeficiente de Pearson $(\mathrm{r}=0,5426)$, concluindo a correlação entre TCEs e a indicação do tratamento clínico em crianças vítimas de acidente motociclístico no Hospital Metropolitano.

Gráfico 2 - Quantitativo de vítimas que sofreram cada tipo de trauma de acidente de motocicleta internadas no HMUE

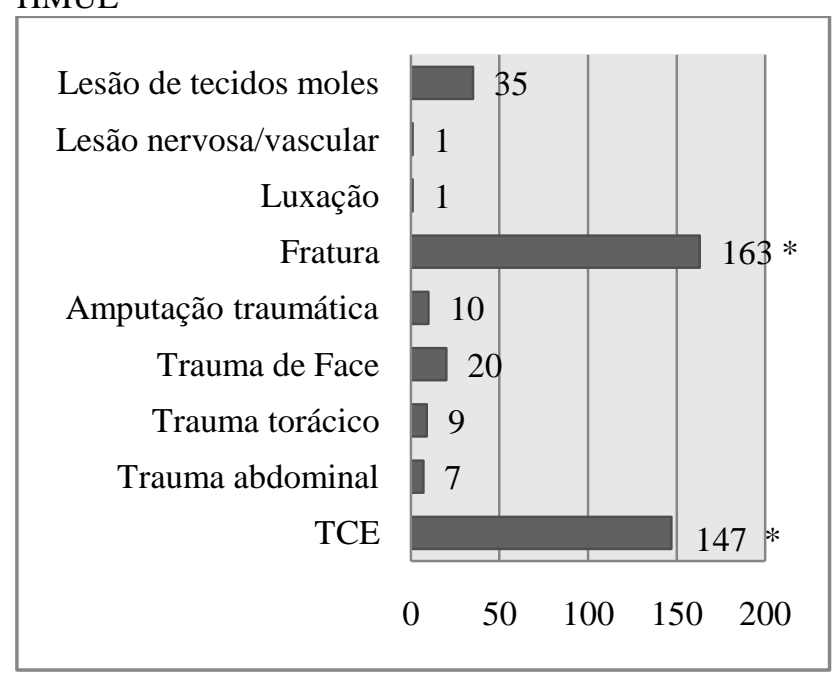

$* \mathrm{p}<0,0001$

Fonte: Dados da Pesquisa

No que consistiu ao número de traumas por indivíduo, verificou-se que a grande maioria com 44, 93\% ( $>>0,0001)$ das crianças estudadas foram internadas por apenas um tipo de trauma. Já 60 delas tiveram lesões em duas estruturas do corpo e 13 apresentaram politraumas em três ou mais regiões do corpo.

Ao analisar as fraturas separadamente, foi possível observar que muitos indivíduos tiveram mais de uma região fraturada, totalizando176 regiões, em 163 indivíduos, sendo as estruturas de joelho e perna as que mais foram lesadas com $n=72$, seguidos de estruturas da coxa $(n=56)$.

Tabela 2 - Distribuição das estruturas acometidas em menores internados vítimas de acidente motociclicleta no HMUE

\begin{tabular}{ccc}
\hline Estrutura & $\mathrm{N}$ & $\%$ \\
\hline Cabeça e pescoço & $170 *$ & 42,82 \\
Membro superior & 35 & 8,81 \\
Pelve & 8 & 2,01 \\
Membro Inferior & $167 *$ & 42,06 \\
Estrutura do tronco & 1 & 0,25 \\
Estrutura da cavidade & 9 & 2,26 \\
$\quad$ abdominal & & \\
Estrutura da cavidade & 7 & 1,76 \\
$\quad$ torácica & & \\
Total & 397 & \\
* p < 0,0001 & & \\
Fonte: Dados da pesquisa & &
\end{tabular}

A maioria das crianças internadas $(n=216)$ realizaram algum tipo de cirurgia (p>0,0001). Por outro lado, das 316 internações, a minoria, 21,2\% das crianças $(n=67)$ estiveram internadas em uma Unidades de Terapia Intensiva do Hospital Metropolitano de Urgência e Emergência ( $p<0,0001)$. Todavia, $12,97 \%$ das crianças, além de necessitarem de cuidados intensivos, fizeram uso de ventilação mecânica invasiva (VMI). Ao analisar a relação em permanência em UTI e necessidade VMI, verifica-se uma correlação considerada forte através do Coeficiente de Pearson: $(r=0,7213)$, induzindo que a grande maioria das crianças que estiveram internadas nas UTIs fizeram uso de ventilação mecânica.

Das crianças que permaneceram internadas no HMUE, 30,69\% (n=97) 
apresentaram algum tipo de complicação ( $p<0,0001)$, muitas dessas, múltiplas. Dentre as complicações, aquelas relacionadas ao sistema respiratório forampredominantes com $\mathrm{n}=35$.

Gráfico 3 - Complicações apresentadas por menores vítimas de acidente motociclísticos durante a internação no HMUE.

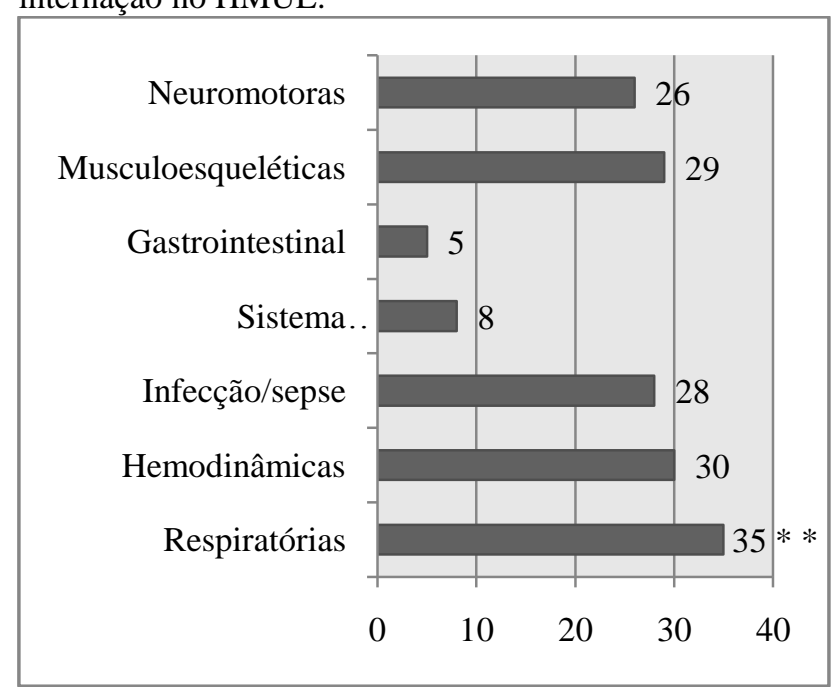

$* * \mathrm{p}<0,0001$

Fonte: Dados da Pesquisa.

Das crianças internadas, apenas $26,26 \%$ realizaram fisioterapia, sendo que $17,72 \%$ realizaram fisioterapia motora $\mathrm{e}$ 20,56\% fisioterapia respiratória.

No que diz repeito ao tempo de internação concluiu-se que houve uma média de 9,74 com desvio padrão (DP) de 11,61. Já em relação às altas, a grande maioria $(97,15 \%)$ teve alta melhorada $(\mathrm{p}<0,0001)$.

Assim, esta pesquisa visou conhecer o perfil de menores até 14 anos, vítimas de acidente envolvendo motocicletas que permaneceram internados no Hospital Metropolitano de Urgência e Emêrgencia no período de 2010 à 2013. Como resultados, foi possível comprovar que as motocicletas são as principais agentes envolvidas nos acidentes de trânsito, entre eles os atropelamentos por esse veículo. Também foi possível observar que houve um crescimento das internações no período estudado sem diferença entre os meses. Dentre os acidentes envolvendo motocicletas, os atropelamentos e os acidentes nos quais ela é conduzida por um menor, ocuparam a grande maioria das internações.

Quanto ao perfil demográfico, crianças de 2 e 14, do gênero masculino, provenientes fora da região metropolitana de Belém anos foram os mais internados. Já no que diz respeito ao perfil clínico, a maioria das crianças traumatizadas apresentaram trauma único e as fraturas e os traumatismos cranioencefálicos foram os principais tipos de trauma, já a cabeça e membros inferiores foram as principais estruturas lesadas. Em grande parte das internações houve necessidade de intervenção cirúrgica, sendo que nas fraturas o principal tratamento foi cirurgico e nos TCE's a maior parte dos pacientes tiveram tratamento conservador. $30,69 \%$ das crianças pesquisadas tiveram algum tipo de complicação, sendo as complicações que concernem ao sistema respiratório a maior partes destas. Também foi possível notar que uma parte pequena porém significante das crianças vítimas de acidentes envolvendo motocicletas 
necessitaram de internação em unidades de terapia intensiva e uma quantidade ainda menor necessitou de ventilação mecânica invasiva.

Observado também que pouco mais de $25 \%$ das crianças atendidas no HMUE neste período realizaram intervenção fisioterapeutica.

Pôde-se notar um tempo médio de internações abaixo do que encontrado em outras pesquisas semelhantes. Também observado um quantitativo baixo de óbitos destas crianças, sendo que a grande maioria teve alta melhorada.

\section{DISCUSSÃO}

Os acidentes de trânsito atingiram um patamar semelhante a outras causas de morbimortalidade no Brasil, como cardiopatias (NUNES; NASCIMENTO, 2010). Por ser um grande problema de saúde também para crianças, muitos estudos têm sido dedicados a este assunto. Os indivíduos da faixa etária jovem, incluindo adolescentes, são associados a maior ocorrência de traumas decorrentes de causas externas e entre os fatores associados a essa maior incidência estão a procura por emoção, o prazer em experimentar situações que o exponham a riscos, inexperiência e a impulsividade(MATOS; MARTINS, 2013).

Entres os tipos de acidente envolvendo motocicletas, podemos observar que as crianças pedestres queforam atingidas por motocicletas e as crianças motociclistas dividem a grande maioria das causas dos acidentes. Com relação ao atropelamento, Wenjun et al. (2011) citam as precárias condições de tráfego, a aplicação insipiente da lei e a baixa segurança no trânsito como fatores que contribuem para fazer os atropelamentos um sério problema em países em desenvolvimento em relação aos países desenvolvidos.

Os dados apresentados neste estudo corroboram com o estudo de Golias e Caetano (2013) que notificaram que entre os acidentes de trânsito, os que envolvem motocicletas representaram $54,2 \%$ de um total de 56.729 acidentes.

Os resultados também convergem com os encontrados no estudo de Andrade e Jorge (2001) que, em sua pesquisa, concluíram que os motociclistas também foram o principal tipo de vítima $(44,4 \%)$ e, quando separados por faixa etária, a população de 1 a 4 anos, apresentou o maior coeficiente de incidência como pedestres, no entanto, na idade de 5 a 14, a maioria eram ciclistas, já a partir de 15 anos, a grande maioria das vítimas eram motociclistas. Resultados semelhantes se observou no levantamento de Gawryzewski et al. (2009), que verificaram que a faixa de zero a 14 anos teve a maioria dos casos classificados como ciclistas, seguido da classificação como pedestres. Assim, pode-se observar que o padrão só se altera na faixa etária de 15 anos em diante, na qual os 
motociclistas são a grande maioria dos acidentados.

Freitas, Ribeiro e Jorge (2007), também notaram que $76,6 \%$ envolveram-se nos acidentes como pedestres ou ciclistas. Estes resultados, são diferentes dos encontrados na presente pesquisa, devido os ciclistas estarem como a segunda categoria de acidentados e não os motociclistas. Esse fato pode ocorrer devido ao período em que ocorreu a coleta de dados nos demais estudos. Sabe-se que cada vez mais a motocicleta está se fazendo presente na realidade de muitos brasileiros e, com o passar dos anos, seu uso está mais difundido, assim sendo, os presentes dados se caracterizaram por informações recentes, acredita-se que pode estar havendo a mudança neste perfil da vítima.

De acordo com a distribuição por sexo, observou-se uma predominância do sexo masculino com idade de 2 a 14 anos. Os indivíduos do sexo masculino também foram os mais envolvidos em acidentes no estudo de Golias e Caetano (2013) e no estudo de Freitas, Ribeiro e Jorge (2007) e isto pode estar relacionadoao fato de eles habitualmente brincarem mais em ambiente extradomiciliar do que meninas.

Gorios et al. (2014) ao descrever o perfil de crianças e adolescentes vítimas de acidentes atendidos em um hospital de São Paulo, apresentaram resultados muito semelhantes com os aqui obtidos, uma vez que as vítimas, em sua maioria, eram do sexo masculino, vítimas de acidentes causados por automóveis ou motocicletas, geralmente, atropelamentos.

É importante que se frise que os resultados também se aproximam de dados de outras pesquisas em diversas partes do mundo, principalmente em países em desenvolvimento. Wenjun et al. (2011) analisaram as ocorrências não fatais de atropelamentos envolvendo crianças na província de Guangdong, China. Os meninos apresentaram uma maior taxa de incidência do que meninas. Já no que consiste a idade, as crianças com idade superior a 10 anos foram as mais expostas aos atropelamentos. Os principais lugares relatados de ocorrências foram calçadas, vias urbanas e faixas de pedestres. O estudo destaca comportamentos de risco das crianças prévios aos acidentes como travessias perigosas e não permitidas e brincadeiras em estradas.

Nwadiaro et al. (2011) também determinaram o padrão de trauma após acidentes graves que envolveram motocicletas na Nigéria, em um Hospital Universitário. Neste levantamento a razão de acidentes envolvendo sexo masculino foi de 4,8 para 1 em relação ao feminino.

Assim, segundo Salvatore apud Freitas, Ribeiro e Jorge (2007) pedestres do sexo masculino fazem julgamentos mais arriscados quanto à velocidade do veículo em aproximação. Pode-se associar a quantidade elevada de acidentes envolvendo homens nos 
acidentes à fatores inerentes ao gênero, mostrando assim o efeito dos padrões socioculturais uma vez que não há fator biológico que determine essa pré-disposição (GOLIAS; CAETANO, 2013).

Apesar do período de declínio das internações, pode-se constatar uma tendência a progressão das internações. Pode-se associar a redução de internações à possível intensificação da fiscalização e da educação no trânsito. Langley, Samaranayaka e Begg (2013) constataramque acidentesmoto ciclísticos aumentaram de 2004 a 2008 na Nova Zelândia em relação ao período precedente estudado (1999 a 2003) e que a população de 10 a 14 anos possui um risco progressivo em se envolver em acidentes de motos atingindo o pico na idade de 15 a 19 anos.

Crianças de 2 anos estarem mais envolvidas em acidentes pode estar relacionado a idade em que estas crianças melhoram suas habilidades motoras e tendem a brincar na rua. Já na pré-adolescência, em menores com idade de 12 a 14 anos ocorrem mudanças importantes no comportamento, assim a própria exigência social de maturidade precoce induz a associação desta faixa etária com acidentes de trânsito, seja como passageiro ou motorista, uma vez que em regiões afastadas há menor policiamento e fiscalização aumentam a possibilidade de ocorrer acidentes seja como motociclista, ciclista ou atropelamento (CEZILLO et al., 2012).

Também podemos observar que a grande maioria das internações precediam de fora da região metropolitana de Belém. Cezillo et al. (2012) referem que as regiões periféricas de centro urbanos apresentam vias de alta velocidade, com fiscalização insipiente e sinalização precária - expondo a riscos de acidentes pedestres e condutores - muitas vezes sem passarelas para atravessia, casas sem espaço para lazer de crianças o que as obrigam a brincar nas ruas, que na maioria das vezes não possuem calçadas, colocando em risco a integridades dos menores.

Com relação ao tipo de trauma, este estudo constatou que as fraturas e os traumatismoscrânioencefálicos

(TCE), somaram o principal tipo de trauma em crianças vítimas de acidentes envolvendo motocicletas. Já no que diz respeito às regiões do corpo atingidas, a cabeça (TCE e traumas de face) e membros inferiores (fraturas, lesões de partes moles, amputações) foram as estruturas mais acometidas.

Os resultados obtidos corroboram com os resultados de Freitas, Ribeiro e Jorge (2007) em que 45,9\% dos indivíduos estudados apresentaram traumatismo crânioencefálico e $\quad 9 \%$ permaneceram internados por mais de duas semanas. Nos resultados de Nwadiaro et al. (2011) o trauma mais frequente também foi em cabeça $(40,1 \%)$ seguido das lesões de extremidades 
$(38,1 \%)$, neste estudo, nenhum dos pacientes usavam capacetes.

O TCE ocorre com frequência em crianças devido sua cabeça ser mais pesada em relação ao restante do corpo, ocorrendo a projeção deste seguimento, ocorrendo com maior gravidade em crianças menores que 3 anos, apresentando assim piores prognósticos (FREITAS, RIBEIRO E JORGE, 2007).

Por outro lado, é observado que há uma alternância entre TCE's e lesões em membros inferiores como principal causa de internação, uma vez que Alicioğluet al. (2008) também analisaram os dados sobre lesões por acidentes de moto na Turquia. Neste estudo porém observou que as lesões do sistema músculo-esquelético foram a maior causa de internações com $(50 \%)$ e a lesões de crânio ocuparam o segundo lugar imediato com 48,6\%, em seguida vieram as lesões maxilofaciais, torácicas, vertebrais e abdominais, respectivamente. Greve et al. (2013), entrevistaram 326 vítimas de acidente de moto afim de avaliar as causas associadas a estes acidentes em São Paulo, neste estudo $17 \%$ tiveram lesões em membros inferiores e apenas 5\% tiveram TCE's associados, que pode ser justificado pelo fato de 90,2\% referirem usar o capacete. No presente estudo, observamos em crianças menores que o uso de capacete, muitas vezes, ser inviável devido ao tamanho desproporcional deste equipamento à cabeça de crianças pequenas.
No estudo de Batista et al. (2012) que avaliou os fatores de risco para fraturas de ossos da face de 393 indivíduos, observou que o acidente de motocicleta éo principal fator de risco para fraturas mandibulares e de um ou mais ossos faciais.

Apesar de não ser a maioria nesta pesquisa, nos casos em que há trauma abdominal com realização deesplenectomia, o risco de sepse por germes capsulados é de $0,026 \%$ por toda a vida com mortalidade de 50\% (CHIARA; CIMBANASSI, 2009). O que comprova a importância de um trauma abdominal, mesmo que único e que este também pode deixar sequelas por toda a vida.

Quando analisada a fratura isoladamente, verificou-se que os membros mais acometidos foram os inferiores com predominância de ossos do joelho e perna, seguido das estruturas da coxa. Esses dados se assemelham com os de Alicioğlu et al. (2008) que concluiu que os membros mais acometidos foram as pernas com fraturas da tíbia - fíbula (17,9\%),no entanto as fraturas no ombro obtiveram o segundo lugar na incidência. Cunha et al. (2007) avaliaram 72 fraturas diafisárias do fêmur sendo o acidente de trânsito o principal responsável pelos traumas, assim observamos a relação importante entre acidentes de transporte e incidência de fraturas.

Como o esqueleto da criança não é totalmente calcificado, esta estrutura não é tão capaz quanto de um adulto de absorver as 
forças cinéticas ocasionadas pelo trauma. É importante que se frise que a criança não é um adulto pequeno, tendo suas peculiaridades e devendo ser tratadas e respeitando-as (MCSWAIN JR et al., 2011).

Segundo Santili et al., (2010), na faixa etária pediátrica, as fraturas de tíbia correspondem de 10 a $15 \%$ de todas e,em crianças e adolescentes, a tíbia é o osso mais fraturado do membro inferior. Quando comparamos a um adulto, observamos a altaprobabilidade de consolidar favoravelmente e em tempo reduzido com tratamento incruento e tendem a evoluir com poucas complicações

Hanna e Austin (2008) constataram que as lesões em membros inferiores foram as mais predominantes em vítimas de acidentes com motocicletas, seguidos pelos traumas em membros superiores e na cabeça, sendo as fraturas mais comuns que lesões de partes moles. Por outro lado, enquanto os traumas em membros inferiores foram mais frequentes, os TCE's, trauma torácico e abdominal tendem a ser mais graves. Em relação ao tipo de alta, os $81 \%$ dos pacientes que sofreram trauma nos membros inferiores foram de alta após cuidado no hospital.

Os traumas que acometem o sistema musculoesquelético, tendem a requerer devida atenção e intervenções terapêuticas em tempo hábil para que não ocorra alterações funcionais por tempo prolongado ou até mesmo permanentemente. Lemos, Jorge e
Ribeiro (2013) comprovam que recursos fisioterapêuticos considerados simples foram suficientes para obter melhores resultados na reabilitação de pacientes vítimas de causas externas. Apesar disso, apenas 26,26\% dos crianças realizaram fisioterapia durante seu período de internação no Hospital Metropolitano de Urgência e Emergência.

A incidência de TCE's de moderado a grave ou depolitraumas, faz necessitar internações em unidades de terapia intensiva e, muitas vezes fazer uso de ventilação mecânica.Maldaun et al. (2002) obtiveram como resultados de uma avaliação dos pacientes vítimas de TCE internados em unidades de terapia intensiva pediátrica, que os atropelamentos foram a principal causa de mecanismo de trauma $(38,5 \%)$. No presente estudo, 21,2 \% dos menores permaneceram internados em uma das unidades de terapia intensiva do HMUE, tais resultados divergem dos obtidos por Freitas, Ribeiro e Jorge (2007) que constataram uma incidência de 7,8\% de internações de crianças em unidades de terapia intensiva devido acidentes de trânsito.

O presente estudo também concluiu que a maior parte das lesões apresentadas por crianças foram traumas únicos, acometendo apenas uma região do corpo, esses resultados corroboram com os dados de Peden et al. (2008) que referem que dos traumas decorrentes de acidente de trânsito em menores, apenas 10 a 20\% são múltiplos. 
Até quando ocorrem pequenos traumas, as crianças menores podem ter a perda de funcionalidade por um período longo, seja na função neurológica, psicologicamente ou em múltiplos sistemas. Cerca de $60 \%$ dos menores vítimas de acidentes que acometeram múltiplos sistemas apresentaram alterações de personalidade e $50 \%$ ficaram com sequelas discretas, tanto físicas como cognitivas (MCSWAIN JRet al., 2011).

No presente estudo, a média de dias internação foi de 9,74 \pm 11,61 dias com apenas $2,85 \%$ de óbitos durante a internação. Estes valores são menores do que os encontrados em Alicioğlu et al. (2008) ao qual a média de internação foi de 12,2 \pm 16,8 dias. Neste, apenas 8 necessitaram de cuidados intensivos em uma média de 7 dias. No mesmo estudo, 4,3\% pacientes evoluíram à óbito devido a acidentes por moto. Ainda a respeito das vítimas, $40 \%$ foram hospitalizadas, e quase $30 \%$ evoluíram com deficiência em longo prazo.

Dados sobre a mortalidade são necessários para que se possa averiguar as condições de saúde e a eficácias das intervenções realizadas, por outro lado,

\section{REFERÊNCIAS}

Alicioğlu B, Yalniz E, Eşkin D, Yilmaz B. Injuries associatedwithmotorcycleaccidents. Acta OrthopTraumatol Turc. 2008 MarApr;42(2):106-11. mesmo quando não ocorre o óbito, deve-se atentar para as sequelas instaladas nas vítimas, que em muitos casos, demandam dedicação e recursos financeiros para sua reabilitação (IMAMURA et al., 2012).

Assim, por meio deste estudo observamos que há uma tendência cada vez maior do uso de motocicleta, e seu fácil acesso e manuseio faz com que indivíduos cada vez mais jovens passem a fazer seu uso. Por outro lado, o Estado deve ser responsável pela educação no trânsito, fiscalização e melhor aplicação da lei, principalmente em regiões mais afastadas dos centros urbanos, para que se evite a grande incidência de acidentes envolvendo crianças e que muitas famílias sejam desestruturadas com tais acidentes.

Por fim, foi possível concluir que os acidentes de motocicletas são uma importante causa de internações em menores, bem como em adultos. No entanto, essa população deve ser estudadaem maneira a parte para que se possa conhecer as características relacionadas a estes acidentes. Este estudo pode servir de embasamento para que se trace estratégias preventivas para reduzir significantemente essas internações.

Andrade SM. Jorge, MHP M. Características das vítimas por acidentes de transporte terrestre em município da Região Sul do Brasil. Rev. Saúde Pública, 2001, 34 (2): 149156 . 
Batista AM, Ferreira FO, Marques LS, Ramos-Jorge ML, Ferreira MC. Braz Oral Res. 2012 Mar-Apr;26(2):119-25.

Cezillo MVB, Müller BR, Lima VP, Adib SCV, Ribeiro RC, AndradeAP. Trauma pediátrico e a questão sociocultural e urbanística na região do Hospital Geral de Pirajussara. Emergência Clínica. 2012, 7 (36):84-91

Chiara O, Cimbanassi S. Protocolo para atendimento intra-hospitalardo trauma grave. Rio de Janeiro: Elsevier, c2009.

Freitas JPP, Ribeiro LA, Jorge MT. Vítimas de acidentes de trânsito na faixa etária pediátrica atendidas em um hospital universitário: aspectos epidemiológicos e clínicos. Cad. Saúde Pública, 2007, Rio de Janeiro, 23(12):3055-3060.

Gawryszewski VP, Coelho HMM, Scarpelini S, Zan R, Jorge MHPM, Rodrigues EMS. Perfil dos atendimentos a acidentes de transporte terrestre por serviços de emergência em São Paulo, 2009. Rev. Saúde Pública; 43( 2 ): 275-282.

Golias ARC, Caetano R. Acidentes entre motocicletas: análise dos casos ocorridos no estado do Paraná entre julho de 2010 e junho de 2011. Ciência\&SaúdeColetiva, 2013, 18 (5):1235-1246

GoriosC,Souza RM, Gerolla V, Maso B, Rodrigues CL, Armond JA, Transport accidents among children and adolescents at the emergency service of a teaching hospital in the southern zone of the city of São Paulo, Rev Bras Ortop . 2014;49(4):391-395

Greve, JMD, Resende MR, Silva HB, Cirino LMI, Bernini CO, Santos JS, Leyton V. Causas de acidentes com motociclistas. São Paulo, 2013. Disponível em:

<http://www.abraciclo.com.br/images/segura nca/pesquisa-causa-de-acidentes-commotocicletas.pdf $>$ acessado em dezembro de 2014.
Hanna R, Austin R. Lower-extremity injuries in motorcycle crashes. Department of Transportation, National Highway Traffic Safety Administration.August, 2008.

Imamura JH. Epidemiologia dos traumas em países desenvolvidos e em desenvolvimento. São Paulo, 2012. 129f. Dissertação (Mestrado em Ciências) - Faculdade de Medicina na Universidade Federal de São Paulo, Universidade Federal de São Paulo, São Paulo, 2012.

Jorge MHPM, Martins CBC. A criança, o adolescente e o trânsito: algumas reflexões importantes. [Editorial] . Rev Assoc Med Bras., 2013, 59 (3) :199-208.

Langley J, Samaranayaka A, Begg, DJ. Age, period and cohort effects on the incidence of motorcyclist casualties in traffic crashes.InjuryPrevention, :153-157, 2013.

Lemos CAG, Jorge MT, Ribeiro LA. Perfil de vítimas e tratamento de lesões por causas externas segundo atendimento pelo Centro de Reabilitacao Municipal de Uberlândia, MG Causas externas e fisioterapia.

RevBrasEpidemiol, 2013; 16(2): 482-92.

Maldaun MVC, Zambelli H JL, Dantas VP, Fabiani RM, Martins AM, Brandão MB. et al . Análise de 52 pacientes com traumatismo de crânio atendidos em UTI pediátrica: considerações sobre o uso da monitorização da pressão intracraniana. Arq. NeuroPsiquiatr. 2002 Dec; 60(4): 967-970.

Mascarenhas MDM, Monteiro RA, Sá NNB, Gonzaga LAA, Neves ACM, Silva MMA, Malta DC. Epidemiologia das causas externas no Brasil: morbidadepor acidentes e violências. Secretaria de Vigilância em Saúde. In: BRASIL, Ministério da Saúde. Secretaria de Vigilância em Saúde. Saúde Brasil 2010: Uma análise da situação de saúde e de evidências, 2013 
Matos KF, Martins CBG. Mortalidade por causas externas em crianças, adolescentes e jovens: uma revisão bibliográfica. Revista Espaço Para a Saúde, 2013, 14(1): 82-93. McSwain Jr NE, Pons PT, Chapleau W, Chapman G, Guy JS, Salomone JP et al. Atendimento Pré Hospitalar ao Traumatizado. 7.ed. Riod de Janeiro: Editora Elsevier, 2011.

Nunes MN, Nascimento LFC. Internações hospitalares por acidentes de moto no vale do paraíba. Rev Assoc Med Bras, Rev Assoc Med Bras. 2010; 56(6): 684-7

Nwadiaro HC, Ekwe KK, Akpayak IC, Shitta $\mathrm{H}$. Motorcycle injuries in north-central Nigeria. Nigerian Journal of Clinical Practice, 2011; 14(2):186-189

Oliveira, NLB; Sousa, RMC. Risk for injuries among motorcyclists involved in traffic incidents. RevEscEnferm USP. 2012 Oct;46(5):1133-40

OxleyJ,Ravi MD, Yuen J, Monash EH, Hashim HH. Identifying contributing factors to fatal and serious injury motorcycle collisions involving children in Malaysia.Ann AdvAutomot Med. 2013 Sep; 57: 329-336.
Peden M, Oyegbite K, Ozanne-Smith J, Hyder AA, Branche C, Rahman AKML, Rivara F, Bartolomeos K. Word report on child injury prevention. Word Health Organization: WHO, 2008.

Pereira LNG, Cancelier ACL, Londero FilhoOM, Franciotti DL, Müller MC, Jornada JK, Avaliação do conhecimento dos pais sobre segurança no transporte de crianças em veículos automotores e motocicletas. Rev Paul Pediatr 2011;29(4):618-24.

Sant'AnnaIFL, Andrade SM, Sant'Anna FHM, LiberattiI CLB. Acidentes com motociclistas: comparação entre os anos 1998 e 2010. Londrina, PR, Brasil. Rev Saúde Pública 2013;47(3):607-15

Santili C, Gomes CMO, AkkariM, Waisberg G, Braga SR, Lino Junior W, Santos FG. Fraturas da diáfise da tíbia em crianças. Acta Ortop Bras. 2010; 18(1):44-8

Wenjun MA, Shaoping, NIE,Haofeng XU, YanjunXU,HuiyanXIE, Y urun Z. Nonfatal Child Pedestrian Injury in Two Urban Cities of Guangdong Province, China : Results from a Cross-sectional Survey. Biomed Environ Sci 2011, 24 (4):335-342. 